\title{
Comparison on Weighing Instrument Calibration between Eramed Kosovo and GDM Albania
}

\author{
Luljeta Disha ${ }^{1}$, Defrim Bulku ${ }^{2}$, Antoneta Deda ${ }^{1}$ \\ ${ }^{1}$ Sector of Mass and Density, General Directorate of Metrology, Albania \\ ${ }^{2}$ Department of Physics, Faculty of Natural Science, Albania
}

\begin{abstract}
The General Directorate of Metrology (GDM) Albania, signed the agreement on collaboration with the Accredited Laboratory ERAMED N.T.SH from Kosovo, to conduct a proficiency testing (PT) in the field of calibration of weghing instrument. Usually testing and calibrating laboratories work with ISO/IEC 17025 standard, one of which requirements is that the laboratories should have quality control procedures for monitoring the validity of tests and calibrations undertaken. It is therefore important that such laboratories should regularly participate in a PT. A weighing instrument with capacity Max $=520 \mathrm{~g}$ and resolution $d=$ $0.1 \mathrm{mg}$ was the subject of this comparison. The measurements were performed on February 2017, at mass laboratory of GDM. This paper describes and analyzes the comparative results and uncertainties associated obtained by this two laboratories. The aim of this comparison is to verify the competence of ERAMED laboratory in the field of calibration of NAWI (non automatic weighing instrument.) The results are analyzed using normalized errors values (En).
\end{abstract}

Keywords: calibration, normalized error, proficiency testing, uncertainty, weighing instrument

\section{Introduction}

The mass laboratory of GDM is the national standard laboratory for mass in Albania. There are realized different Key Comparisons in which mass laboratory of GDM was involved, in the framework of IPA 2008 project supported by European Commission and some other supported by European Association of National Metrology Institutes (EURAMET). The degrees of equivalence resulting from these comparisons are within their respective standard uncertainties showing that the Key Comparison was a success for mass laboratory in Albania. Participation in a proficiency testing can validate the participating laboratory's measurement method, technical training, traceability of standards, and uncertainty budgets. The subject of this bilateral comparison was an electronic weighing instrument with $\mathrm{Max}=520 \mathrm{~g}$ and resolution $\mathrm{d}=0.1 \mathrm{mg}$. This instrument has not been transported. Both laboratories agreed to perform the measurements at mass laboratory in Albania. This paper reports the results obtained by two laboratories in a proficiency testing scheme. The aim of the comparison was not only to compare measurement results of the participant with those of Pilot laboratory, but also to analyze measurement uncertainty, the choice of calibration points and to check the validity of quoted calibration measurement capabilities (CMC). The result is considered successful, if the value of the normalized error is $-1 \leq$ $E n \leq 1$. In this case the participating laboratory agrees with the reference value within the stated uncertainty. This paper is of interest for accredited or non-accredited laboratories for the calibration of NAWI as well for accreditation bodies.

\section{MEASUREMENT INSTRUCTION}

Initially, ERAMED mass laboratory of Kosovo announced its participation in the comparison of weighing instrument calibration and the mass laboratory of GDM accepted the role as the Pilot laboratory. Calibration will be performed over the full weighing range from zero to the maximum capacity. The test loads used for determining the errors of indication were: $0 \mathrm{~g}, 100 \mathrm{~g}, 200 \mathrm{~g}, 300 \mathrm{~g}, 400 \mathrm{~g}$ and $500 \mathrm{~g}$. Metrological and technical characteristics of the weighing instrument were provided before starting the proficiency testing [1]. Participants will use their standards procedures of calibration for which they will be validated and claim their CMC. The errors of indication and uncertainty associated to these errors were determined according to [2]. For acclimatization, it was proposed that ERAMED mass laboratory brings in advance the weights at mass laboratory in Albania. Standards weights used for calibration by Pilot laboratory have uncertainty according to CMC. The comparison measurements were carried out on February 2017 and the time schedule is reported in Table 1. 
Table 1 Time schedule of comparison

\begin{tabular}{|c|c|c|c|}
\hline Time - start & Time - end & $\begin{array}{c}\text { Time periods } \\
\text { Working days }\end{array}$ & Action \\
\hline 01 February 2017 & 14 February 2017 & 14 days & $\begin{array}{c}\text { Preparation of technical protocol PL } \\
\text { GDM }\end{array}$ \\
\hline 14 February 2017 & 15 February 2017 & 2days & $\begin{array}{c}\text { Measurements from ERAMED } \\
\text { Laboratory }\end{array}$ \\
\hline 16 February 2017 & 22 February 2017 & 7 days & Measurements from Pilot Laboratory \\
\hline 24 February 2017 & 30 April 2017 & 35 days & $\begin{array}{c}\text { Analyses of results and final report from } \\
\text { Pilot Laboratory }\end{array}$ \\
\hline
\end{tabular}

In order to be in the same conditions of calibration performance, both laboratories will do the adjustment of the weighing instrument before calibration according to the user manual.

For the calibration of the balance the Pilot laboratory used direct comparison method with standard weights having nominal mass and accuracy class presented in the Table 2 . The standard weights used by ERAMED laboratory are shown in Table 3

Table 2 Standard weights used for calibration by GDM mass laboratory

\begin{tabular}{|c|c|c|c|c|c|c|}
\hline $\begin{array}{c}\text { Nominal } \\
\text { value }\end{array}$ & Manufacturer & $\begin{array}{c}\text { Serial } \\
\text { Number }\end{array}$ & Identification & Class & Uncertainty (U) & Traceability /Certificate \\
\hline $1 \mathrm{~g}-500 \mathrm{~g}$ & HAIGIS & 7259 & LM-07/03 & E2 & $(4.0-95) \mu \mathrm{g}$ & LM-1116G-223 GDM \\
\hline
\end{tabular}

Table 3 Standard weights used for calibration by ERAMED mass laboratory

\begin{tabular}{|c|c|c|c|c|c|c|}
\hline Nominal value & Manufacturer & $\begin{array}{c}\text { Serial } \\
\text { Number }\end{array}$ & Identification & Class & $\begin{array}{c}\text { Uncertainty } \\
\text { (U) }\end{array}$ & $\begin{array}{c}\text { Traceability/ } \\
\text { Certificate }\end{array}$ \\
\hline $1 \mathrm{mg}-1 \mathrm{~kg}$ & SARTORIUS & 26629505 & Inv. Nr-026 & E2 & $(3.0-500) \mu \mathrm{g}$ & $\begin{array}{c}\text { BoM Macedonia } \\
12.2016\end{array}$ \\
\hline
\end{tabular}

Standards weights used for calibration by Pilot Laboratory have the form and accuracy according to [3] and are traceable to the primary mass laboratory of GDM. Repeatability was determined at 500g and 200g. For each load, ten measurements were performed. The test of eccentricity is performed at $200 \mathrm{~g}$. The error of indication and uncertainty associated to these errors were determined according to equation (1) and (2) respectively. For loading the balance, both laboratories have opted to load the balance only once, increasing, by steps with unloading between the separate steps, corresponding to the majority of uses of the instruments for weighing single loads.

$$
E_{j}=I_{j}-m_{\text {ref }}
$$

$\mathrm{Ij}$ - the indication of the balance

$\mathrm{m}_{\text {ref }}$ - is conventional mass of the reference weights

$$
U(E)=2 \mathrm{u}(\mathrm{E})=\sqrt{\frac{\mathrm{d}_{0}^{2}}{12}++\frac{\mathrm{d}_{\mathrm{L}}^{2}}{12}+\mathrm{s}^{2}(\mathrm{I})+\mathrm{u}_{\mathrm{ecc}}^{2}+\mathrm{u}^{2}\left(\mathrm{~m}_{\mathrm{ref}}\right)}
$$

The signification of the terms from the formula (2) is explained in Fig.1 [4].

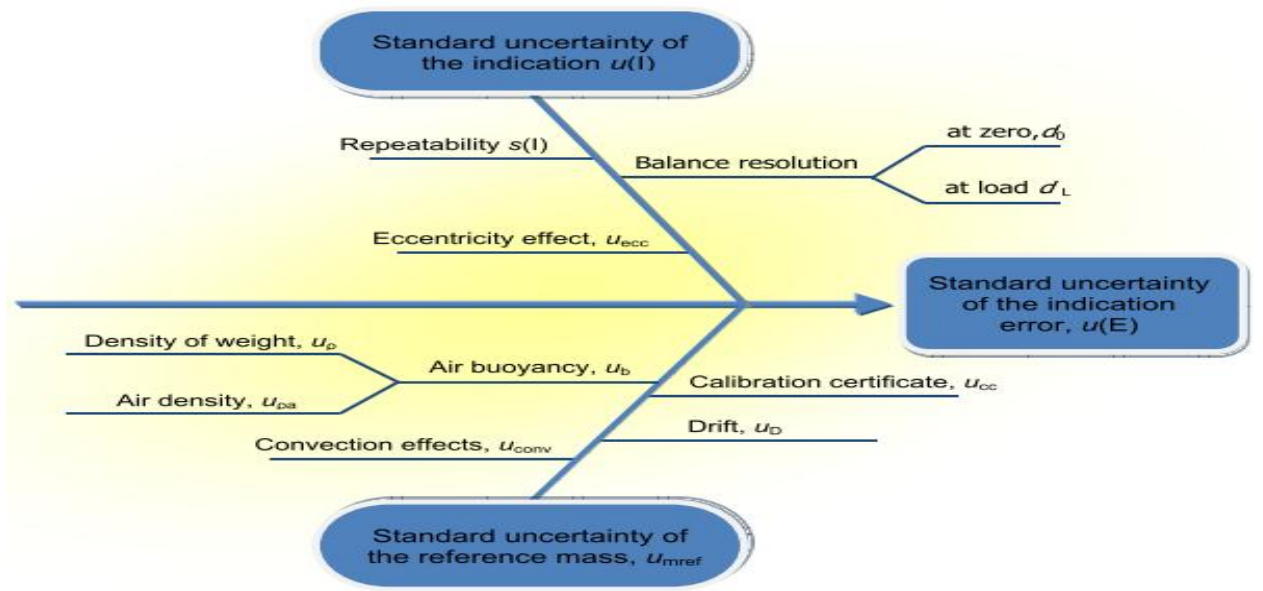

Figure1 Ishikawa diagram of uncertainty components in the calibration of the balance 


\section{Analyses And Results Of Comparison}

After completion of the measurements, the measurements results of participant laboratory were sent to the scheme coordinator in the required format of the proficiency test protocol, in a period of 7 working days. Then, the Pilot laboratory performed the analysys of results. Consistency of the test is considered satisfactory when the probably test of the results for participant $\chi^{2}$ is fulfilled [5]

According to the analyses of Pilot laboratory the condition $\operatorname{Pr}\left\{\chi^{2}(v)>\chi_{\text {obs }}^{2}\right\}<0.05$ is fulfied for each load. Depending on the evaluation procedure used, the reference value is defined as the weighted mean of Pilot laboratory measurements according to equation (3) [5].

$$
\Delta \mathrm{m}_{\text {ref }}=\frac{\sum_{\mathrm{i}=1}^{\mathrm{N}} \frac{\Delta \mathrm{m}_{\mathrm{c}-\mathrm{P}}}{\mathrm{u}\left(\Delta \mathrm{m}_{\mathrm{c}-\mathrm{P}}\right)^{2}}}{\sum_{\mathrm{i}=1}^{\mathrm{N}} \frac{1}{\mathrm{u}\left(\Delta \mathrm{m}_{\mathrm{c}-\mathrm{P}}\right)^{2}}}
$$

where $\overline{\Delta \mathrm{m}}_{\mathrm{c}}=\Delta \mathrm{m}_{\mathrm{c}}=\operatorname{Ref}$

In order to carry out consistency checks of the results the uncertainty in the reference value has been calculated also according to the methods described by [5] with equation (4).

$$
\mathrm{u}\left(\Delta \mathrm{m}_{\mathrm{c}-\mathrm{ref}}\right)=\frac{1}{\sum_{\mathrm{i}=1}^{\mathrm{N}} \frac{1}{\mathrm{u}\left(\Delta \mathrm{m}_{\mathrm{c}-\mathrm{P}}\right)^{2}}}
$$

where: $U_{\left(\overline{\Delta m_{\mathrm{w}}}\right)}=\mathrm{U}_{\left(\Delta \mathrm{m}_{\mathrm{ref}}\right)}=\mathrm{U}_{\mathrm{Ref}}$

In Table 4 below are presented all the components of uncertainty reported by the participant laboratory and by the Pilot laboratory.

\begin{tabular}{|c|c|c|c|}
\hline \multicolumn{4}{|c|}{ (Standard) uncertainty contributions (mg) } \\
\hline Uncertainty component & Symbol & GDM & ERAMED \\
\hline Repeatability & $(s)$ & $\mathrm{x}$ & $\mathrm{x}$ \\
\hline Resolution (0 load) & $u\left(R_{0}\right)$ & $\mathrm{x}$ & $\mathrm{x}$ \\
\hline Resolution (L load) & $u\left(R_{L}\right)$ & $\mathrm{x}$ & $\mathrm{x}$ \\
\hline Uncertainty due to eccentricity effect & $u\left(\Delta_{\text {mecc }}\right)$ & $\mathrm{x}$ & $\mathrm{x}$ \\
\hline Uncertainty of the indication & $u(I)$ & $\mathrm{x}$ & $\mathrm{x}$ \\
\hline Uncertainty due to mass standards & $u\left(\Delta_{m c}\right)$ & $\mathrm{x}$ & $\mathrm{x}$ \\
\hline Uncertainty due to drift of mass standards & $u\left(\Delta_{m D}\right)$ & $\mathrm{x}$ & $\mathrm{x}$ \\
\hline Uncertainty due to buoyancy correction & $u\left(\Delta_{m B}\right)$ & $\mathrm{x}$ & $\mathrm{x}$ \\
\hline Uncertainty due to convection effect & $u\left(\Delta_{\text {mconv }}\right)$ & $\mathrm{x}$ & - \\
\hline Standard uncertainty of the error of indication $u_{\mathrm{c}}(k=1)$ & $u(E)$ & $\mathrm{x}$ & $\mathrm{x}$ \\
\hline
\end{tabular}

Table 4 The ccomponents of uncertainty reported by ERAMED and Pilot laboratory

Table 5 presents the errors of indication " $E$ ", calculated according to formula (1) and the expanded uncertainty " $U "(\mathrm{k}=2)$ associated with these errors, calculated according to formula (2), reported by Pilot Laboratory and ERAMED laboratory

Table 5 The errors of indication "E" and expanded uncertainty "U" reported by GDM and ERAMED laboratory

\begin{tabular}{|c|c|c|c|c|}
\hline \multirow{2}{*}{$\begin{array}{c}\text { Load } \\
(\mathrm{g})\end{array}$} & \multicolumn{3}{|c|}{ Laboratory } \\
\cline { 2 - 5 } & $\mathrm{E}(\mathrm{g})$ & $\mathrm{U}(\mathrm{g})$ & $\mathrm{E}(\mathrm{g})$ & $\mathrm{U}(\mathrm{g})$ \\
\hline 0 & 0.00000 & 0.00010 & 0.00000 & 0.00012 \\
\hline 100 & 0.00050 & 0.00011 & 0.00060 & 0.00024 \\
\hline 200 & 0.00109 & 0.00013 & 0.00120 & 0.00042 \\
\hline 300 & 0.00159 & 0.00018 & 0.00180 & 0.00095 \\
\hline 400 & 0.00223 & 0.00022 & 0.00250 & 0.00123 \\
\hline 500 & 0.00310 & 0.00026 & 0.00360 & 0.00164 \\
\hline
\end{tabular}

\subsection{Normalized errors of the reported values and degrees of equivalence for participant laboratory}

A tool often used in analyzing the results from interlaboratory comparisons is the normalized error $E$ n, which takes into account both the result and its uncertainty. The normalized error is calculated according to equation (5) [5]:

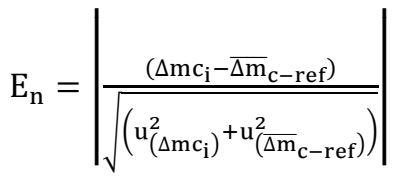


The result is considered successful, if the value of the normalized error is $-1 \leq E \mathrm{n} \leq 1$. In Table 6 are presented the normalized errors $E$ n of the reported values and degrees of equivalence for participant laboratory. In this table the term $\Delta \mathrm{m}_{\mathrm{c}}\left(\mathrm{deq}_{\mathrm{A}}\right)$ is calculated as difference between participant values and pilot laboratory reference values and expresses the degree of equivalence for participant laboratory. In the other hand, $\Delta U\left(\mathrm{deq}_{\mathrm{A}}\right)$ is the term related to uncertainty and calculated also as a difference against reference uncertainty value.

Table 6 Normalized errors $E$ n and degrees of equivalence for participant laboratory

\begin{tabular}{|c|c|c|c|}
\hline Nominal value & $\mathrm{E}_{\mathrm{n}}$ ERAMED & $\Delta \mathrm{m}_{\mathrm{c}}\left(\mathrm{deq}_{\mathrm{A}}\right)(\mathrm{g})$ & $\Delta \mathrm{U}\left(\mathrm{deq}_{\mathrm{A}}\right)(\mathrm{g})$ \\
\hline $500 \mathrm{~g}$ & 0.301 & 0.00050 & 0.00162 \\
\hline $400 \mathrm{~g}$ & 0.216 & 0.00027 & 0.00121 \\
\hline $300 \mathrm{~g}$ & 0.217 & 0.00021 & 0.00093 \\
\hline $200 \mathrm{~g}$ & 0.250 & 0.00011 & 0.00040 \\
\hline $100 \mathrm{~g}$ & 0.379 & 0.00010 & 0.00021 \\
\hline $0 \mathrm{~g}$ & 0.000 & 0.00000 & 0.00007 \\
\hline
\end{tabular}

From Table 6 it visible that the values of normalized error are lowers than 1 for participant laboratory and in this case it can be said that the measurements results of participant laboratory are consistent with the reference values. Figure 2 presents the reference values of Pilot laboratory and participant measurements results with corresponding uncertainties.

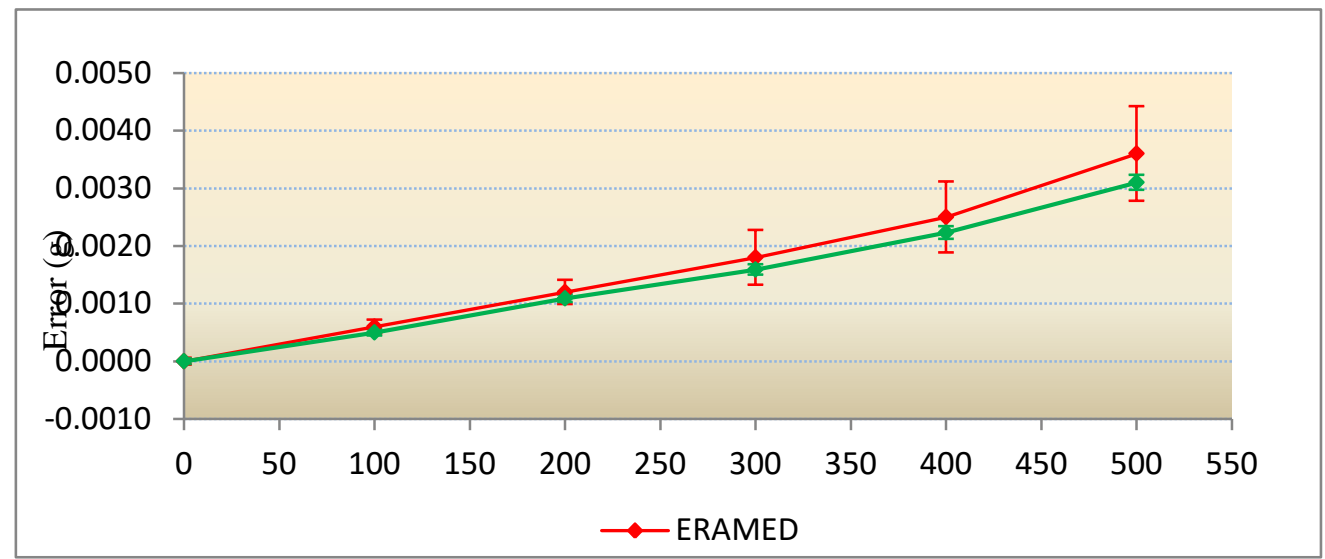

Figure 2 Reference values of Pilot laboratory and participant measurements results with corresponding uncertainties

\section{Conclusions}

A bilateral comparison in the field of calibration of weighing instruments between GDM, Albania and ERAMED, Kosovo was performed. Calibration laboratories announced their participation in a comparison of a NAWI, having Max 520g and $\mathrm{d}=0.1 \mathrm{mg}$. In this case the participating laboratory agrees with the reference value within the stated uncertainty. If this laboratory has any unsuccessful results, i.e. $E \mathrm{n}<-1$ or $E \mathrm{n}>1$, it is expected that the laboratory investigates the reason for the disagreement and implements corrective action. From this point of view, it can be concluded that bilateral comparison between the GDM mass laboratory and ERAMED mass laboratory was successful. Consequently the performance of the mass laboratory ERAMED was satisfactory, validating in this way its technical competence in the field of calibration of weighing instruments. In Albania, Proficiency Testing in the field of calibration of non automatic weighing instruments is a new subject.

\section{References}

[1] OIML Recommendation R 76-1 "General requirements for metrological and technical characteristics" of NAWI. 2006

[2] EURAMET, cg -18 "Guidelines on the Calibration of Non-automatic Weighing Instruments", Version $4.0 / 2015$

[3] Weights of classes E1, E2, F1, F2, M1, M1-2, M2, M2-3 and M3, International recommendation OIML R111-1, OIML, (Paris), 2004

[4] Adriana Valcu, "Between Parallel Mirrors - An Electronic Weighing Instrument", Proc. 9th International symposium on advanced topics in electrical engineering, Bucuresti, 2015.

[5] Cox M. G. 2002, Metrologia 39 589-595

Luljeta Disha. "Comparison on Weighing Instrument Calibration between Eramed Kosovo and GDM

Albania." IOSR Journal of Applied Physics (IOSR-JAP) 9.4 (2017): 60-63. 